\title{
Geographic Variation in the Sensitivity of Recombinant Antigen-based Rapid Tests for Chronic Trypanosoma cruzi Infection
}

\author{
Jennifer R. Verani,* Amy Seitz, Robert H. Gilman, Carlos LaFuente, Gerson Galdos-Cardenas, \\ Vivian Kawai, Elizabeth de LaFuente, Lisbeth Ferrufino, Natalie M. Bowman, Viviana Pinedo-Cancino, \\ Michael Z. Levy, Francis Steurer, Charles W. Todd, Louis V. Kirchhoff, Lilia Cabrera, \\ Manuela Verastegui, and Caryn Bern \\ Division of Parasitic Diseases, Centers for Disease Control and Prevention, Atlanta, Georgia; Emory University, Atlanta, Georgia; \\ Johns Hopkins University Bloomberg School of Public Health, Baltimore, Maryland; Asociación Benéfica PRISMA, Lima, Peru; \\ Universidad Peruana Cayetano Heredia, Lima, Peru; Hospital Unversitario Japonés, Santa Cruz, Bolivia; Fogarty International Center, \\ National Institutes of Health, Bethesda, Maryland; Carver College of Medicine, University of Iowa, Iowa City, Iowa
}

\begin{abstract}
Chagas disease affects 8-11 million people throughout the Americas. Early detection is crucial for timely treatment and to prevent non-vectorial transmission. Recombinant antigen-based rapid tests had high sensitivity and specificity in laboratory evaluations, but no Peruvian specimens were included in previous studies. We evaluated Stat-Pak and Trypanosoma Detect rapid tests in specimens from Bolivia and Peru. Specimens positive by three conventional assays were confirmed positives; specimens negative by two or more assays were confirmed negatives. In Bolivian specimens, Stat-Pak and Trypanosoma Detect tests were $87.5 \%$ and $90.7 \%$ sensitive, respectively; both showed $100 \%$ specificity. Sensitivity in Peruvian specimens was much lower: 26.6-33.0\% (Stat-Pak) and 54.3-55.2\% (Trypanosoma Detect); both had specificities $>98 \%$. Even in Bolivian specimens, these sensitivities are inadequate for stand-alone screening. The low sensitivity in Peru may be related to parasite strain differences. Chagas disease rapid tests should be field tested in each geographic site before widespread implementation for screening.
\end{abstract}

\section{INTRODUCTION}

Chagas disease, caused by the parasite Trypanosoma cruzi, affects an estimated 8-11 million people throughout the Americas, and > 100 million people live in areas where they are at risk of becoming infected. ${ }^{1-3}$ Of those infected, as many as $30 \%$ develop debilitating - and potentially lifethreatening - cardiac and/or gastrointestinal disease. ${ }^{4}$ People become infected when the T. cruzi-containing feces of the blood-sucking triatomine vector contaminate the bite wound or mucous membranes. Non-vectorial transmission may occur through blood transfusion, organ transplantation, laboratory accident, or congenitally. Without successful treatment, infection is life long. Therapy with currently available antitrypanosomal drugs is associated with reversion to negative serology in $50-60 \%$ of treated children ${ }^{5,6}$ and may decrease the likelihood of clinical progression of Chagas cardiomyopathy in adults. ${ }^{7}$ Early detection is crucial for timely treatment and to prevent non-vectorial transmission.

Conventional serologic tests, including the indirect hemagglutination assay (IHA), enzyme-linked immunosorbent assay (ELISA), and immunofluorescent antibody test (IFA), are commonly used to diagnose chronic Chagas disease. Because no single test has optimal sensitivity and specificity, the World Health Organization recommends the use in parallel of at least two distinct serologic assays to confirm T. cruzi infection. ${ }^{1}$ Epimastigote lysate-based ELISAs tend to have the highest sensitivity but have substantial rates of cross-reaction with other infections such as leishmaniasis..$^{8,9}$ Furthermore, conventional assays require specialized laboratory equipment and trained laboratory personnel, and testing may require several days. Rapid tests based on recombinant antigens have been developed in an effort to improve the specificity of Chagas testing and to offer a simple diagnostic technique that provides rapid results. One commercially available rapid test

* Address correspondence to Jennifer R. Verani, 4770 Buford Highway MS F-22, Atlanta, GA 30341. E-mail: jverani@cdc.gov based on several recombinant antigens, the Stat-Pak (Chembio Diagnostic Systems, Medford, NY), showed high sensitivity and specificity in laboratory testing of specimens from several endemic countries, including Bolivia. ${ }^{10,11}$ However, no specimens from Peru were included in the studies published to date. At the time we undertook this study, no studies on the performance of other rapid tests and no field evaluation of Stat-Pak had been published. To assess the utility of these tests for use in field studies, we evaluated the performance of two Chagas disease rapid tests in serum specimens from Peru and Bolivia.

\section{MATERIALS AND METHODS}

The specimens included in this analysis were collected during two epidemiologic studies of Chagas disease. The Bolivian specimens were tested in the study hospital laboratory by a technician used by the research project following the manufacturer's instructions. Because the performance of the rapid tests in Peru was entirely unknown, the Peru specimen evaluation followed a stringently timed protocol, including readings by two independent observers, and was conducted in the Parasitic Diseases Serology Laboratory at the Centers for Disease Control and Prevention (CDC), Atlanta, Georgia. Both study protocols were approved by the Institutional Review Boards of the CDC, Johns Hopkins University Bloomberg School of Public Health, and Asociación Benéfica Proyectos en Informática, Salud, Medicina y Agricultura (PRISMA), Lima, Peru. Additional ethical approvals were obtained from the Hospital Universitario Japonés, Santa Cruz, Bolivia, for the study in Bolivia and the Instituto Nacional de Salud, Lima, Peru, for the Peru study.

Source and evaluation of Bolivian specimens. The Bolivian specimens were collected during recruitment for a study of congenital Chagas disease transmission in the Hospital Universitario Japonés, Santa Cruz, Bolivia, conducted from November 2006 to June 2007. Women presenting in labor to the obstetrical service of the hospital were asked to participate in the first step of the study, the aim of which was to 
identify women with $T$. cruzi infection at risk of transmission to their infants. After informed consent, a 5-mL blood specimen was collected from each woman, centrifuged in the hospital laboratory, and separated into clot and several aliquots of serum. The study laboratory technician used one fresh serum aliquot to perform the Stat-Pak and Trypanosoma Detect (InBios International, Seattle, WA) rapid tests in the hospital laboratory within a few hours of collection, following the manufacturer's instructions. The rapid tests were used before their expiration dates. The Stat-Pak tests were donated by the Bolivian National Chagas Disease Control program and were used with consecutive specimens until the supply ran out; therefore, Stat-Pak testing was available for a subset of specimens only. The Trypanosoma Detect, which was not commercially available, was a prototype (ITC-8.2) provided by the manufacturer.

One aliquot of each serum specimen was frozen without additives and shipped from Bolivia to the Infectious Disease Laboratory at the Universidad Peruana Cayetano Heredia in Lima, Peru, where an in-house IFA following standard methods ${ }^{12}$ and two commercially available ELISAs (Chagatek ELISA; bioMérieux, Marcy l'Etoile, France [based on epimastigote lysate antigen] and Chagatest Recombinante ELISA [recombinant proteins]; Wiener Laboratories, Rosario, Argentina) were performed on all specimens. Following the manufacturer's instructions for the Chagatek plates, the cut-off was set at 0.100 optical density units (OD) above the mean absorbance of the two negative control specimens included on each plate. For the Wiener plates, the cut-off was set at 0.300 OD units above the mean absorbance of three negative control specimens; specimens with OD values within the range of the cut-off $\pm 10 \%$ were considered inconclusive and were re-run. If the OD still fell in this range, the final result was considered inconclusive. Specimens positive or negative by all three conventional serologic tests were considered to have confirmed results. Specimens with discordant or inconclusive results were excluded.

Source and evaluation of Peruvian specimens. The Peruvian field site comprised two districts on the outskirts of the city of Arequipa where recent vector-borne T. cruzi transmission had been documented. ${ }^{13-15}$ Household-level insecticide spray campaigns were carried out by the Ministry of Health in late 2004; a serosurvey for Chagas disease was conducted in NovemberDecember 2005. ${ }^{15}$ Most participants were recruited in the public schools serving the two districts. In addition, the entire population of one hillside shantytown was invited to enroll in a community-based study. ${ }^{13}$ School age children living in the shantytown were recruited either in schools or in the community. A 5 -mL blood specimen $(3 \mathrm{~mL}$ for children $<5$ years of age) was collected from each participant, separated into serum and cells, aliquoted into cryovials, and frozen without additives in the laboratory of the Universidad Nacional de San Agustín in Arequipa. Specimens were shipped on dry ice to Lima, Peru, for further processing.

All Peruvian specimens were tested by the same T. cruzi epimastigote lysate-based ELISA kit (Chagatek), as for Bolivian samples. Frozen aliquots of all ELISA-positive specimens plus a $10 \%$ random sample of ELISA-negative specimens were shipped on dry ice to CDC in Atlanta, and tested by an in-house IFA using standard methods; a titer of 1:32 was considered the threshold for a positive result. ${ }^{12}$ All specimens positive by ELISA or IFA, plus $20 \%$ of the negative specimens tested at $\mathrm{CDC}$, were frozen and shipped on dry ice to the laboratory of one of the authors (LVK), where they were tested by the radioimmune precipitation assay (RIPA). The RIPA is often used in the United States to confirm positive results obtained in other tests and to resolve the status of specimens with discordant conventional tests. ${ }^{16,17}$ Specimens tested by all three assays (at least two assays for ELISA-negative specimens) were eligible for inclusion in this analysis. Specimens positive by all three assays (ELISA, IFA, and RIPA) were considered confirmed positive. Not all specimens negative by ELISA and IFA were tested by RIPA, because of the high cost of this assay; specimens were considered confirmed negative if they had negative results in the two (ELISA and IFA) or three assays (ELISA, IFA, and RIPA) used.

Confirmed positive and negative specimens were tested by the Stat-Pak and Trypanosoma Detect rapid tests in the CDC laboratory. For both tests, the manufacturer's instructions were rigorously followed. Each test was read at 15 minutes based on a laboratory timer by two independent observers blinded to each other's reading. The presence of a weak or strong line in the reaction zone was recorded as positive; its absence was recorded as negative. Readers also recorded comments, such as "weak positive," "very weak positive," "very faint," or "unreadable." For sensitivity/specificity analysis, interpretations of "positive," "weak positive," "very weak positive," and "very faint" were all considered positive. Specimens with "unreadable" rapid test results excluded from analysis.

Data analysis. Sensitivities and specificities with $95 \%$ confidence intervals (CIs) and weighted $\kappa$ coefficients for interobserver agreement were calculated using SAS V9.1 and SAS Enterprise Guide V4.1 (SAS Institute, Cary, NC). The Wilcoxon rank-sum test was used to compare differences for Chagatek ELISA absorbance (as measured by OD values minus cut-off value) for positive specimens, stratified by country of origin and rapid test results (true positive versus false negative).

\section{RESULTS}

Characteristics of the study populations, specimens, and testing are summarized in Table 1 . In both cases, the case definition for confirmed positive specimens required positive results on three conventional serologic tests.

Bolivian field assessment. Of 530 specimens from women presenting to give birth in the hospital in Santa Cruz, 151 (28.4\%) were confirmed positive and 365 (68.9\%) were confirmed negative by all three conventional tests (Chagatek ELISA, Wiener ELISA, and IFA). Fourteen (2.6\%) specimens with discordant (nine) or inconclusive (five) serologic results were excluded from the analysis. The Trypanosoma Detect was positive in 137 of the 151 confirmed positive sera and negative in all 365 confirmed negative sera, corresponding to a sensitivity of $90.7 \%$ and specificity of $100 \%$ (Table 2). The Stat-Pak was positive in 21 of 24 confirmed positive sera and negative in all 69 confirmed negative sera tested, yielding a sensitivity of $87.5 \%$ and specificity of $100 \%$.

Six Stat-Pak readings carried the comment "weak positive" and one "very weak positive," representing 30\% of all positive readings; all were considered positive following the manufacturer's instructions, and all were confirmed positive in the conventional tests. Two specimens had Stat-Pak-negative readings at 15 minutes but "very weak positive" at 25-30 minutes; based on the manufacturer's instructions, these readings 
TABLE 1

Comparison of characteristics of the specimens from Bolivia and Peru included in this analysis

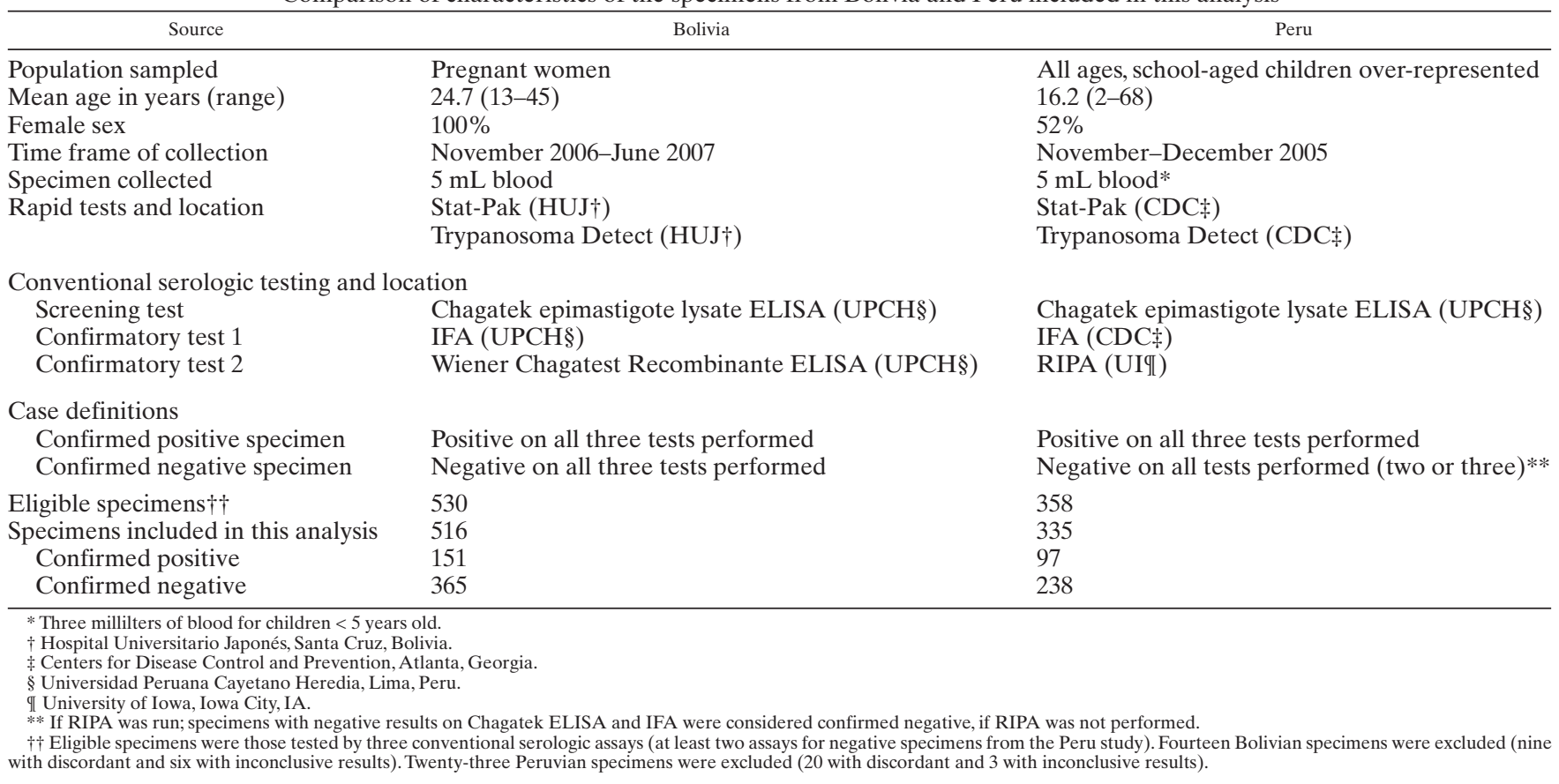

were considered negative. One of these specimens was confirmed positive and the other negative based on the three conventional tests. Fifteen Trypanosoma Detect readings, or 10\% of positive readings, were recorded as "weak positive," three of which were also "weak positive" on Stat-Pak. All were positive on the three conventional serologic tests.

Evaluation in Peruvian specimens. The serosurvey in Peru included 2,254 specimens, of which $120(5.3 \%)$ were positive by the screening ELISA. Of these, 364 (120 positive and 244 negative by the screening ELISA) were shipped to CDC for confirmatory testing. At CDC, $102(85.0 \%)$ of 120 ELISApositive specimens and $5(2.0 \%)$ of 244 ELISA-negative specimens were positive by IFA. Four ELISA-positive specimens lacked sufficient remaining volume to perform RIPA. RIPA results were therefore available for 98 of the 102 specimens positive by ELISA and IFA; 97 (99.0\%) were positive, whereas $1(1.0 \%)$ had an indeterminate RIPA result and was excluded from further analysis. Of 240 specimens negative by ELISA and IFA, 48 were tested by RIPA; 46 (95.8\%) were negative. One $(2.1 \%)$ was positive by RIPA, and one $(2.1 \%)$ had an indeterminate result; these specimens also were excluded from further analysis. The group of specimens used to evaluate rapid test performance therefore consisted of 97 specimens positive by ELISA, IFA, and RIPA and 238 specimens

TABLE 2

Performance of two rapid tests in specimens from Santa Cruz, Bolivia*

\begin{tabular}{|c|c|c|c|c|}
\hline \multirow{2}{*}{$\begin{array}{l}\text { Conventional } \\
\text { serology }\end{array}$} & \multicolumn{2}{|c|}{ Chembio Stat-Pak } & \multicolumn{2}{|c|}{ InBios Trypanosoma Detec } \\
\hline & Negative & Positive & Negative & Positive \\
\hline Negative & 69 & 0 & 365 & 0 \\
\hline Positive & 3 & 21 & 14 & 137 \\
\hline Sensitivity $\dagger$ & \multicolumn{2}{|c|}{$87.5(67.6-97.3)$} & \multicolumn{2}{|c|}{90.7 (84.9-94.8) } \\
\hline Specificity $\dagger$ & \multicolumn{2}{|c|}{$100(94.8-100)$} & \multicolumn{2}{|c|}{$100(99.0-100)$} \\
\hline
\end{tabular}

*A lysate-based ELISA, a recombinant ELISA, and radioimmune precipitation assay were used to determine the final status of the specimens.

$\dagger$ Sensitivity or specificity expressed as a percentage and $95 \% \mathrm{CI}$ negative by ELISA and IFA, 46 of which also were confirmed negative by RIPA (Table 1 ).

The observers reading the rapid tests in Peruvian specimens reported many weak and indeterminate readings for both tests. Although the tests are intended to be read as "positive" or "negative," 100 (59\%) of the 169 positive test readings from two readers carried notations of "weak positive," "very weak positive," or "very faint" on one or both rapid tests (Figure 1). Weighted $\kappa$ coefficient for interobserver agreement was 0.760 (95\% CI: 0.652-0.867) for Stat-Pak, reflecting "substantial" agreement between readers and 0.833 (95\% CI: 0.780-0.885) for Trypanosoma Detect, or "almost perfect" agreement between readers. The Stat-Pak showed sensitivities of $26.6 \%$ by Observer 1 and $33.0 \%$ by Observer 2; the specificity by both readers was $99.6 \%$ (Table 3 ). Trypanosoma Detect sensitivities were $53.8 \%$ and $54.6 \%$; specificities were $98.7 \%$ and $98.3 \%$.
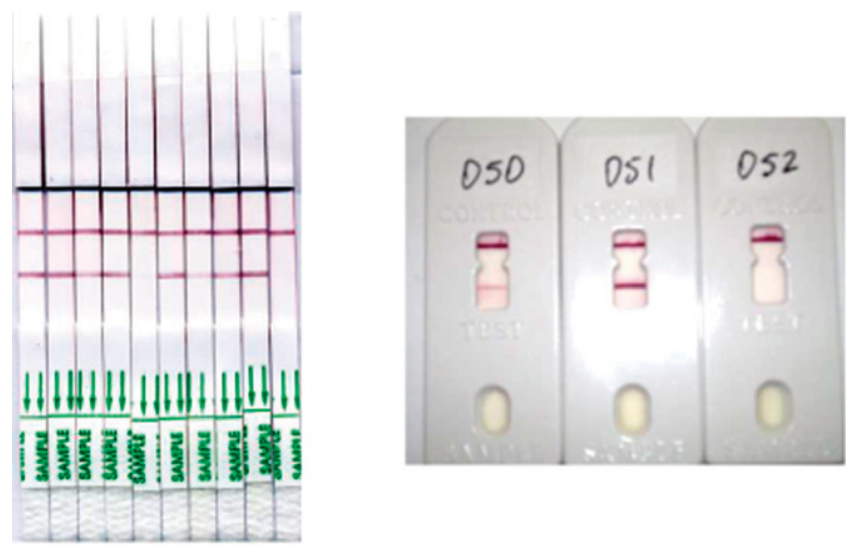

Figure 1. The InBios Trypanosoma Detect (left) and Chembio Chagas Stat-Pak (right) rapid tests. Note the weak positive results in each of the tests. This figure appears in color at www.ajtmh.org. 
TABLE 3

Performance of two rapid tests in specimens from Arequipa, Peru*

\begin{tabular}{|c|c|c|c|c|c|c|c|c|}
\hline & \multicolumn{4}{|c|}{ Chembio Stat-Pak } & \multicolumn{4}{|c|}{ InBios Trypanosoma Detect } \\
\hline & \multicolumn{2}{|c|}{ Observer 1} & \multicolumn{2}{|c|}{ Observer 2} & \multicolumn{2}{|c|}{ Observer 1} & \multicolumn{2}{|c|}{ Observer 2} \\
\hline & Negative & Positive & Negative & Positive & Negative & Positive & Negative & Positive \\
\hline Negative & 237 & 1 & 237 & 1 & 235 & 3 & 234 & 4 \\
\hline Positive & 69 & 25 & 65 & 32 & 43 & 50 & 44 & 53 \\
\hline Sensitivity $\dagger$ & \multicolumn{2}{|c|}{$26.6(18.0-36.7)$} & \multicolumn{2}{|c|}{$33.0(23.8-43.3)$} & \multicolumn{2}{|c|}{$53.8(43.1-64.2)$} & \multicolumn{2}{|c|}{$54.6(44.2-64.8)$} \\
\hline Specificity $\dagger$ & \multicolumn{2}{|c|}{$99.6(97.7-100)$} & \multicolumn{2}{|c|}{$99.6(97.7-100)$} & \multicolumn{2}{|c|}{98.7 (96.4-99.7) } & \multicolumn{2}{|c|}{$98.3(95.7-99.5)$} \\
\hline
\end{tabular}

Each test was read by two independent observers.

* A lysate-based ELISA, a recombinant ELISA, and radioimmune precipitation assay were used to determine the final status of the specimens.

$\dagger$ Sensitivity or specificity expressed as a percentage and $95 \%$ CI.

ELISA results. The ELISA absorbance among confirmed positive specimens was significantly higher in Bolivian than Peruvian specimens (0.842 versus $0.305 ; P<0.0001$; Figure 2$)$. For Trypanosoma Detect, Bolivian specimens with truepositive results had a median absorbance of 0.873 versus 0.203 for false negatives $(P<0.0001)$; in Peruvian specimens, the respective values were 0.394 and $0.195(P<0.0001$; Figure 3$)$. For Stat-Pak, the median for Bolivian specimens with truepositive results was 0.831 compared with 0.122 for false negatives $(P=0.08)$; in Peruvian specimens, the respective values were 0.470 and $0.254(P<0.05$; Figure 4$)$.

\section{DISCUSSION}

Our evaluation of the Chembio Stat-Pak and InBios Trypanosoma Detect rapid tests in specimens from Bolivia and Peru showed highly variable sensitivities. In Peruvian specimens, both rapid tests had sensitivities $<60 \%$, making them unusable in this setting in their current forms. Although the sensitivities in Bolivian specimens were much higher than in Peruvian samples, they still fell short of previously published values, ${ }^{10,11,18}$ missing $10 \%$ or more of specimens confirmed positive by conventional serology. In both locations, the ELISA absorbance values were significantly higher among specimens with true-positive rapid test results than among those with false-negative results, suggesting that the rapid test detection

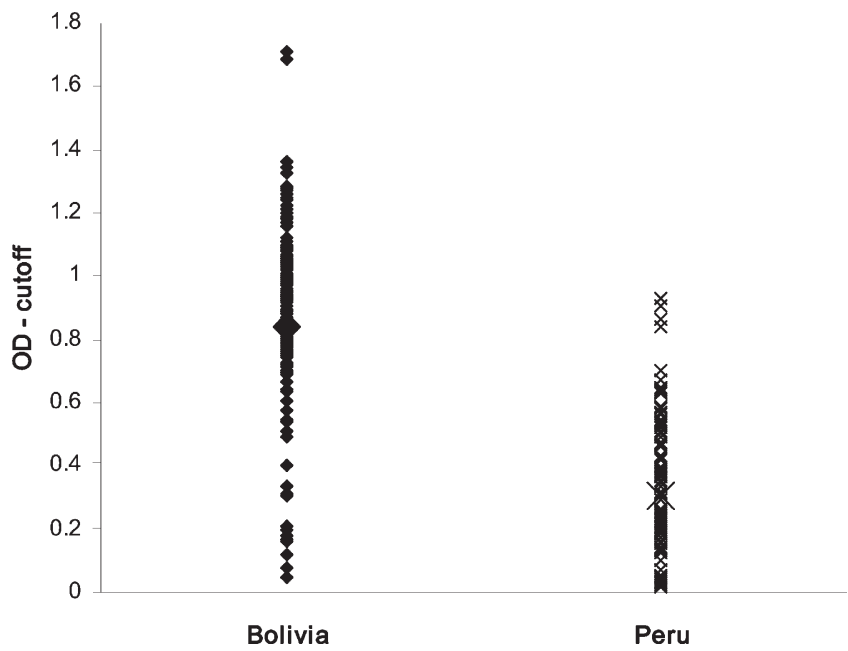

Figure 2. Distribution and median of Chagatek ELISA absorbance (OD minus cut-off value) among confirmed positive samples in studies in Bolivia and Peru. Confirmed positive specimens were defined as those with positive results on all three conventional serologic tests (Chagatek ELISA, IFA, and either Wiener Recombinant ELISA [Bolivia] or RIPA [Peru]). thresholds may be set too high. In Peru, many rapid test readings were noted as "very weak" and therefore difficult to interpret. Nevertheless, interobserver agreement was high. The specificities determined in specimens from both locations were acceptably high.

The poor sensitivity of the Stat-Pak test, particularly in the Arequipa specimens, contrasts sharply with previously published data on its performance. One evaluation of Stat-Pak showed sensitivities of $98.5 \%$ in 393 Brazilian specimens and $100 \%$ in 352 specimens from Honduras, Argentina, Venezuela, and Bolivia. ${ }^{10}$ An evaluation of the Stat-Pak in nearly 6,000 blood bank specimens from Honduras, El Salvador, and Nicaragua showed sensitivity of $99.6 \% .{ }^{11}$ The Stat-Pak was found to have a somewhat lower sensitivity of $93.4 \%$ in a recent field study in Sucre, Bolivia. ${ }^{18}$ The authors hypothesized that the lower sensitivity resulted from the use of whole blood instead of serum; however, no results using serum from the same study subjects were provided to address this issue. Another recently published study from southern Bolivia reported a $5.9 \%$ discordance rate between Stat-Pak and an epimastigote lysate-based ELISA (Wiener Chagatest), but no details were provided regarding the rate of false-positive and false-negative results. ${ }^{19}$

Our findings suggest that there may be more regional variation in antibody response to T. cruzi infection than is usually acknowledged. ${ }^{20}$ In a study using ELISAs based on each of

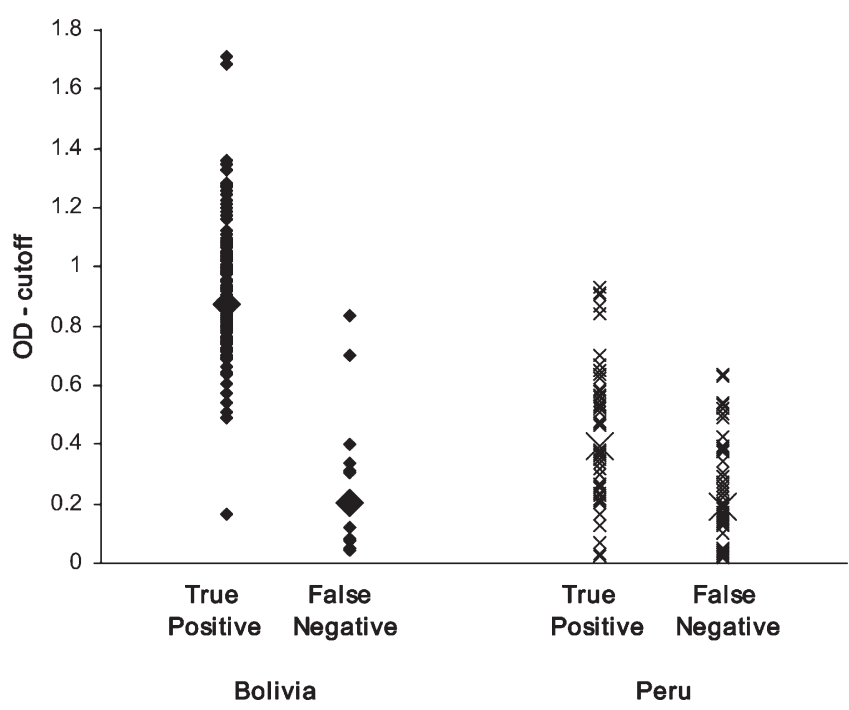

Figure 3. Distribution and median of Chagatek ELISA absorbance (OD minus cut-off value) of confirmed positive samples with true-positive and false-negative results by Trypanosoma Detect rapid test in studies in Bolivia and Peru. 


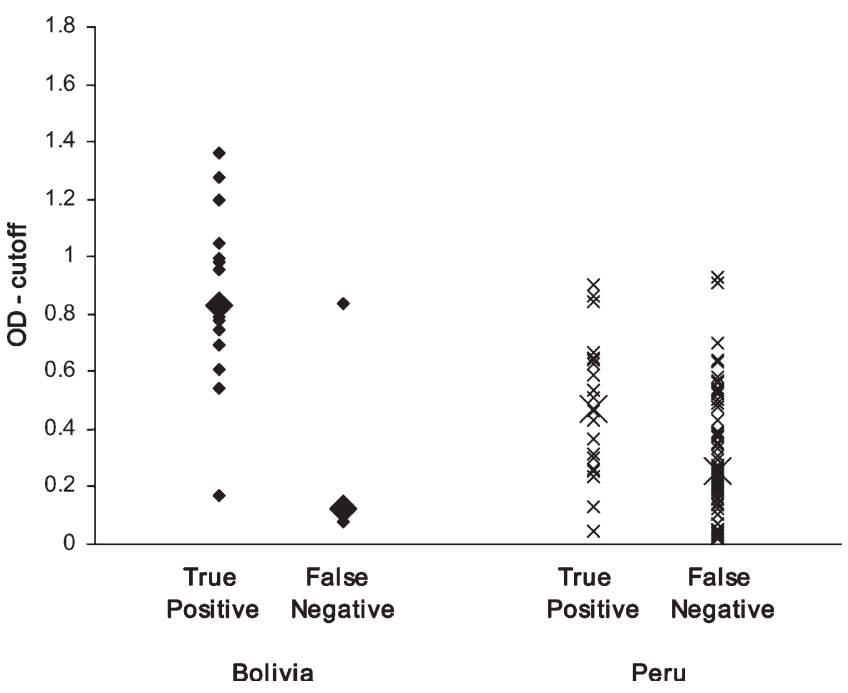

FIGURE 4. Distribution and median of Chagatek ELISA absorbance (OD minus cut-off value) of confirmed positive samples with true-positive and false-negative results by Stat-Pak rapid test in studies in Bolivia and Peru.

six recombinant $T$. cruzi antigens (including at least three of the antigens used in the Stat-Pak) to test sera from Argentina, Bolivia, Brazil, Chile, Colombia, El Salvador, Guatemala, Honduras, and Venezuela, Umezawa and others ${ }^{21}$ found that the reactivity of individual antigens varied by geographic region. Although a whole epimastigote-lysate-based ELISA had a sensitivity of $100 \%$, individual antigens reacted with $79-100 \%$ of seropositive samples from the various countries. Another analysis showed consistently lower absorbance values for some recombinant antigens in specimens from Panama compared with Bolivia, Brazil, and Honduras. ${ }^{22}$ We found much lower epimastigote lysate ELISA absorbance values in Peru compared with Bolivia, suggesting a less robust antibody response in Peru. Because the populations of Santa Cruz and Arequipa are fairly comparable mixes of European and Native American descendants, human genetic factors are unlikely to explain this major difference in immunologic response. We suspect that the variable performance of these two rapid tests may be caused by parasite heterogeneity. The species T. cruzi includes several strains with heterogeneous genetic and phenotypic characteristics, ${ }^{23}$ but the implications for use of recombinant antigens for serologic diagnosis are as yet undetermined. There are no published evaluations of Chagas rapid test performance or of the performance of recombinant antigen-based ELISA kits in Peruvian specimens, and our data raise questions about the assumption that these two rapid tests will perform similarly in the various regions of Latin America in which Chagas disease is endemic.

This study had a number of limitations. The samples were collected in a limited geographic area within each country, and our results cannot be generalized to other locations. The epidemiologic methods and objectives were different in the two studies, and the distributions by age and sex were therefore different (Table 1). However, there are no data to suggest that the sensitivity of rapid tests should vary greatly between males and females or among school-aged children compared with pregnant women. Because both populations are prime targets for Chagas disease screening in current control programs, it is important that any screening test be equally effective for both.
We were unable to use the same second confirmatory test (RIPA) in Bolivian as Peruvian specimens because of resource constraints. Nevertheless, we believe that our definition of confirmed positive was equally stringent for both sets of specimens. Indeed, the use of three tests may have excluded positive specimens with lower antibody levels and thereby resulted in an overestimate of the true sensitivity of the rapid tests. The only methodologic difference that might contribute to a lower sensitivity in the Peru specimens was the fact that they had been through two freeze-thaw cycles before the rapid tests were used, whereas the Bolivian specimens were tested fresh. However, RIPA testing of the Peruvian specimens occurred after a third freeze-thaw cycle and showed high concordance with the screening ELISA. In any case, two freeze-thaw cycles should not cause a $50-70 \%$ decrease in the sensitivity of a robust serologic test. Clearly, further evaluations of rapid test performance in a broader range of populations and under a variety of conditions are still needed.

Rapid tests for Chagas disease have the potential to facilitate both prenatal screening and surveys to detect infected children who would benefit from drug treatment. To date, the challenging logistics of community and hospital-based screening and follow-up have prevented these efforts from reaching large proportions of the population in need. ${ }^{24}$ The Stat-Pak and Trypanosoma Detect rapid tests are simple to use and require no refrigeration, specialized equipment, or highly trained laboratory personnel. The results are available within 15 minutes, eliminating the need to return a second time to a community or to track down an infected woman after discharge from the hospital. The use of recombinant antigens rather than whole epimastigote preparations may improve the specificity of Chagas testing, particularly in areas where Chagas disease and leishmaniasis are co-endemic. These attributes make rapid tests very appealing for use in field epidemiology and clinical diagnostics. Stat-Pak has been used for community-based screening of children in Bolivia, ${ }^{25}$ and its use has been advocated for other settings where conventional serology is not readily available. ${ }^{10,11}$

However, the specific antigens and format used in rapid tests may result in varying sensitivity depending on the location in which the test is applied; Arequipa seems to be one such location for which these rapid tests are not well adapted. Furthermore, rapid test sensitivity in Bolivian specimens was low for use as the sole screening tool. A child or pregnant woman with a false-negative rapid test may not ever be tested again, but a false-positive rapid test result will be detected when confirmatory serology is run. Thus, higher sensitivity would be worth the trade-off of lower specificity that would inevitably result from lowering the detection threshold. Until consistently high sensitivity can be shown, rapid diagnostic tests should be carefully evaluated in specimens from the intended target locations before widespread implementation, and screening of new populations should continue to include testing by sensitive conventional assays in all specimens.

Received June 20,2008. Accepted for publication October 20, 2008.

Acknowledgments: The authors thank C. Espada, E. Hinojosa, R. J. Ledezma, N. Selum, and S. Quispe in Santa Cruz; A. Saman, E. Ramos, N. Figueroa, J. Calderon, P. Smith, C. Mendoza Gleny, R. Villazante, K. del Carpio, S. Quispe, JG. Cornejo del Carpio, E Cordova-B F Delgado, and J. Pastor in Arequipa; P. Maguiña, P. Herrera, and M. Meza at AB PRISMA; and C. Salazar at Johns Hopkins Bloomberg School of Public Health. Stat-Pak tests used in the Peru evaluation 
were donated by Chembio Diagnostic Systems; Stat-Pak tests for the Bolivian evaluation were donated by the Bolivian National Chagas Disease Control Program. Trypanosoma Detect tests for both evaluations were donated by InBios International.

Financial support: This work was supported by NIH P50 AI074285-01, NIH 1R21 AI072093-01, and NIH K01 AI079162-01.

Disclosure: Dr. Kirchoff serves as a consultant for Abbott Laboratories, receives payments through licensing agreements from the latter and from Quest Diagnostics, and owns an equity interest in Goldfinch Diagnostics. This statement is made in the interest of full disclosure and not because the authors consider this to be a conflict of interest.

Authors' addresses: Jennifer R. Verani, Amy Seitz, Robert H. Gilman, Carlos LaFuente, Gerson Galdos-Cardenas, Vivian Kawai, Elizabeth de LaFuente, Lisbeth Ferrufino, Natalie M. Bowman, Viviana PinedoCancino, Michael Z. Levy, Francis Steurer, Charles W. Todd, Louis V. Kirchhoff, Lilia Cabrera, Manuela Verastegui, and Caryn Bern, Centers for Disease Control and Prevention, 1600 Clifton Road NE, MS C-23, Atlanta, GA 30333.

\section{REFERENCES}

1. World Health Organization, 2002. Control of Chagas disease. World Health Organ Tech Rep Ser 905: i-vi, 1-109.

2. Organizacion Panamericana de la Salud, 2006. Estimación cuantitativa de la Enfermedad de Chagas en las Amèricas. OPS/OMS, ed. OPS/HDM/CD/425-06. Montevideo: OPS/OMS.

3. Remme JHF, Feenstra P, Lever PR, Medici A, Morel C, Noma M, Ramaiah KD, Richards F, Seketelli A, Schmunis G, Brakel WH, Vassal A, 2006. Tropical diseases targeted for elimination: Chagas disease, lymphatic filariasis, onchocerciasis, and leprosy. Jamison DT, Breman JG, Measham AR, Alleyne G, Claeson M, Evans DB, Jha P, Mills A, Musgrove P, eds. Disease Control Priorities in Developing Countries. New York: The World Bank and Oxford University Press, 433-449.

4. Maguire J, 2004. Trypanosoma. Gorbach SL BJ, Blacklow NR, eds. Infectious Diseases. Philadelphia: Lippincott, Williams and Wilkins, 2327-2334.

5. de Andrade AL, Zicker F, de Oliveira RM, Almeida Silva S, Luquetti A, Travassos LR, Almeida IC, de Andrade SS, de Andrade JG, Martelli CM,1996. Randomised trial of efficacy of benznidazole in treatment of early Trypanosoma cruzi infection. Lancet 348: 1407-1413.

6. Sosa Estani S, Segura EL, Ruiz AM, Velazquez E, Porcel BM, Yampotis C, 1998. Efficacy of chemotherapy with benznidazole in children in the indeterminate phase of Chagas' disease. Am J Trop Med Hyg 59: 526-529.

7. Viotti R, Vigliano C, Lococo B, Bertocchi G, Petti M, Alvarez MG, Postan M, Armenti A, 2006. Long-term cardiac outcomes of treating chronic Chagas disease with benznidazole versus no treatment: a nonrandomized trial. Ann Intern Med 144: 724-734.

8. Caballero ZC, Sousa OE, Marques WP, Saez-Alquezar A, Umezawa ES, 2007. Evaluation of serological tests to identify Trypanosoma cruzi infection in humans and determine crossreactivity with Trypanosoma rangeli and Leishmania spp. Clin Vaccine Immunol 14: 1045-1049.

9. Malchiodi EL, Chiaramonte MG, Taranto NJ, Zwirner NW, Margni RA, 1994. Cross-reactivity studies and differential serodiagnosis of human infections caused by Trypanosoma cruzi $i$ and Leishmania spp; use of immunoblotting and ELISA with a purified antigen (Ag163B6). Clin Exp Immunol 97: $417-423$.

10. Luquetti AO, Ponce C, Ponce E, Esfandiari J, Schijman A, Revollo S, Anez N, Zingales B, Ramgel-Aldao R, Gonzalez A, Levin MJ, Umezawa ES, Franco da Silveira J, 2003. Chagas' disease diagnosis: a multicentric evaluation of Chagas Stat-Pak, a rapid immunochromatographic assay with recombinant proteins of Trypanosoma cruzi. Diagn Microbiol Infect Dis 46: 265-271.
11. Ponce C, Ponce E, Vinelli E, Montoya A, de Aguilar V, Gonzalez A, Zingales B, Rangel-Aldao R, Levin MJ, Esfandiari J, Umezawa ES, Luquetti AO, da Silveira JF, 2005. Validation of a rapid and reliable test for diagnosis of Chagas' disease by detection of Trypanosoma cruzi-specific antibodies in blood of donors and patients in Central America. J Clin Microbiol 43: $5065-5068$.

12. Camargo ME, Rebonato C, 1969. Cross-reactivity in fluorescence tests for Trypanosoma and Leishmania antibodies. A simple inhibition procedure to ensure specific results. Am J Trop Med Hyg 18: 500-505.

13. Levy MZ, Bowman NM, Kawai V, Waller LA, Cornejo del Carpio JG, Cordova Benzaquen E, Gilman RH, Bern C, 2006. Periurban Trypanosoma cruzi-infected Triatoma infestans, Arequipa, Peru. Emerg Infect Dis 12: 1345-1352.

14. Levy MZ, Kawai V, Bowman NM, Waller LA, Cabrera L, PinedoCancino VV, Seitz AE, Steurer FJ, Cornejo Del Carpio JG, Cordova-Benzaquen E, Maguire JH, Gilman RH, Bern C, 2007. Targeted screening strategies to detect Trypanosoma cruzi infection in children. PLoS Negl Trop Dis 1: e103.

15. Bowman NM, Kawai V, Levy MZ, Cornejo del Carpio JG, Cabrera L, Delgado F, Malaga F, Cordova Benzaquin E, Pinedo VV, Steurer F, Seitz A, Gilman R, Bern C, 2008. Chagas disease transmission in peri-urban communities of Arequipa, Peru. Clin Infect Dis 46: 1822-1828.

16. Leiby DA, Wendel S, Takaoka DT, Fachini RM, Oliveira LC, Tibbals MA, 2000. Serologic testing for Trypanosoma cruzi: comparison of radioimmunoprecipitation assay with commercially available indirect immunofluorescence assay, indirect hemagglutination assay, and enzyme-linked immunosorbent assay kits. J Clin Microbiol 38: 639-642.

17. Kirchhoff LV, Gam AA, Gusmao RA, Goldsmith RS, Rezende JM, Rassi A, 1987. Increased specificity of serodiagnosis of Chagas' disease by detection of antibody to the 72- and 90kilodalton glycoproteins of Trypanosoma cruzi. J Infect Dis 155: 561-564.

18. Roddy P, Goiri J, Flevaud L, Palma PP, Morote S, Lima N, Villa L, Torrico F, Albajar-Vinas P, 2008. Field evaluation of a rapid immunochromatographic assay for detection of Trypanosoma cruzi infection by use of whole blood. J Clin Microbiol 46: 2022-2027.

19. Chippaux JP, Postigo JR, Santalla JA, Schneider D, Brutus L, 2008. Epidemiological evaluation of Chagas disease in a rural area of southern Bolivia. Trans R Soc Trop Med Hyg 102: 578-584.

20. Kirchhoff LV, Paredes P, Lomeli-Guerrero A, Paredes-Espinoza M, Ron-Guerrero CS, Delgado-Mejia M, Pena-Munoz JG, 2006. Transfusion-associated Chagas disease (American trypanosomiasis) in Mexico: implications for transfusion medicine in the United States. Transfusion 46: 298-304.

21. Umezawa ES, Bastos SF, Camargo ME, Yamauchi LM, Santos MR, Gonzalez A, Zingales B, Levin MJ, Sousa O, Rangel-Aldao R, da Silveira JF, 1999. Evaluation of recombinant antigens for serodiagnosis of Chagas' disease in South and Central America. J Clin Microbiol 37: 1554-1560.

22. Umezawa ES, Luquetti AO, Levitus G, Ponce C, Ponce E, Henriquez D, Revollo S, Espinoza B, Sousa O, Khan B, da Silveira JF, 2004. Serodiagnosis of chronic and acute Chagas' disease with Trypanosoma cruzi recombinant proteins: results of a collaborative study in six Latin American countries. J Clin Microbiol 42: 449-452.

23. Campbell DA, Westenberger SJ, Sturm NR, 2004. The determinants of Chagas disease: connecting parasite and host genetics. Curr Mol Med 4: 549-562.

24. Blanco SB, Segura EL, Cura EN, Chuit R, Tulian L, Flores I, Garbarino G, Villalonga JF, Gurtler RE, 2000. Congenital transmission of Trypanosoma cruzi: an operational outline for detecting and treating infected infants in north-western Argentina. Trop Med Int Health 5: 293-301.

25. Chippaux JP, Postigo JR, Santalla JA, Schneider D, Brutus L, 2008. Epidemiological evaluation of Chagas disease in a rural area of southern Bolivia. Trans R Soc Trop Med Hyg 102: 578-584. 